Marquette University

e-Publications@Marquette

Mechanical Engineering Faculty Research and

Publications

Mechanical Engineering, Department of

7-6-2004

\title{
Admittance Selection for Force-guided Assembly of Polyhedral Parts in Single-point Contact
}

Shuguang Huang

Marquette University, shuguang.huang@marquette.edu

Joseph M. Schimmels

Marquette University, joseph.schimmels@marquette.edu

Accepted version. IEEE International Conference on Robotics and Automation, 2004. Proceedings. ICRA '04. 2004, Accession Number: 8082491 (July 6, 2004). DOI. C 2018 IEEE. Used with permission. 
Marquette University

e-Publications@Marquette

\title{
Mechanical Engineering Faculty Research and Publications/College of Engineering
}

This paper is NOT THE PUBLISHED VERSION; but the author's final, peer-reviewed manuscript. The published version may be accessed by following the link in the citation below.

IEEE International Conference on Robotics and Automation, 2004. Proceedings. ICRA '04. 2004, (26 April-1 May 2004). DOI. This article is (C) Institute of Electrical and Electronic Engineers (IEEE) and permission has been granted for this version to appear in e-Publications@Marquette. Institute of Electrical and Electronic Engineers (IEEE) does not grant permission for this article to be further copied/distributed or hosted elsewhere without the express permission from Institute of Electrical and Electronic Engineers (IEEE).

\section{Admittance Selection for Force-guided Assembly of Polyhedral Parts in Single-point Contact}

\section{Shuguang Huang}

Department of Mechanical and Industrial Engineering, Marquette University, Milwaukee, WI J.M. Schimmels

Department of Mechanical and Industrial Engineering, Marquette University, Milwaukee, WI

\begin{abstract}
:
The selection of the proper admittance is important in achieving force-guided assembly. This paper identifies procedures for selecting the appropriate spatial admittance to achieve reliable force-guided assembly of polyhedral parts for single-point frictionless contact cases. Sets of conditions that are imposed on the admittance matrix for different types of single-point contact are presented. These conditions ensure that the motion that results from contact reduces part misalignment in the selected contact state. We show that, for bounded misalignments, if an admittance satisfies the misalignment-reduction conditions at a finite number of contact configurations, then the admittance also satisfy the conditions at all intermediate configurations.
\end{abstract}




\section{SECTION I. Introduction}

In robotic assembly tasks, the manipulator admittance can provide force regulation and force guidance. An admittance is a mapping of forces into velocities. To achieve reliable assembly, the manipulator admittance must be appropriate for the particular assembly task.

We consider a simple form of admittance, a linear admittance control law. ${ }^{1}$ For spatial applications. this admittance behavior has the form:

$$
\mathrm{v}=\mathrm{v}_{0}+\mathbf{A w}(1)
$$

where $v_{0}$ is the nominal twist (6-vector), $w$ is the contact wrench (force and torque) measured in the body frame (a 6-vector), $A$ is the admittance matrix (a $6 \times 6$ matrix), and $v$ is the motion of the body.

Admittance design for force-guidance has been addressed by many researchers. Whitney ${ }^{2,3}$ proposed that the compliance of a manipulator be structured so that contact forces lead to decreasing errors.

Peshkin ${ }^{4}$ addressed the synthesis of an accommodation (inverse damping) matrix by specifying the desired force/motion relation at a sampled set of positional errors for a planar assembly task. Others ${ }^{5,6}$ provided synthesis procedures based on spatial intuitive reasoning.

A reliable admittance selection approach is to design the control law so that, at each possible part misalignment, the contact force always leads to a motion that reduces the existing misalignment. The approach is referred to as force-assembly and has been successful for workpart into fixture insertion when errors are infinitesimal. ${ }^{1,7,8}$

In previous work, ${ }^{9,10,11}$ sufficient conditions for an admittance to ensure force-guided assembly for planar polygonal parts have been identified. In this paper, sufficient conditions for an admittance to ensure force-guidance for spatial polyhedral parts is identified. Due to the nature of motion, the kinematic description of a spatial polyhedral part is significantly different than that of a planar polygonal part.

Consistent with prior work, we consider a measure of error based on the Euclidean distance between an arbitrarily chosen single (fixed) point on the held body and its location when properly positioned. Because the selection of the reference location is arbitrary, one configuration dependent location (point of maximum distance) can be selected to use an established metric ${ }^{12}$ or more than one location can be selected to further restrict the description of what constitutes error-reduction in rigid body assembly.

The misalignment reduction condition of force-assembly requires that, at each possible misalignment, the contact force yields a motion that reduces the misalignment. Using the point-based measure of misalignment discussed above, this condition can be expressed mathematically if we let $d$ (a 6-vector for spatial motion) be the line vector from the selected point at its properly mated position to its current position. Then, for error reducing motion, the condition is: 


$$
\mathrm{d}^{T} \mathrm{v}=\mathrm{d}^{T}\left(\mathrm{v}_{0}+\mathbf{A w}\right)<0(2)
$$

which must be satisfied for all possible misalignments.

Because the line vector $d$ depends on the rigid body configuration and because the number of configurations is infinite, it is impossible to impose the error-reduction condition for all misalignments. In application, however, the misalignments of the rigid body are bounded by: 1) the extremes within a contact state, or 2) the extremes of manipulator, inaccuracy, Those misalignments at the "extremes" are of particular interest.

This paper considers polybedral rigid body assembly involving spatial motion constrained by frictionless contact. The contact states studied here are the non-degenerate, single-point principal contacts (or $\mathrm{PCS})^{13}$ obtained for polyhedral parts.

Polyhedral bodies in single-point contact have three types of stable principle contacts: "face-vertex" ( $\{f-$ $v\}))$ contact, "vertex-face" $(\{v-f\})$ contact, and "edge-edge cross" $\left(\{e-e\}_{c}\right)$ contact. Each of the single-point principle contacts are illustrated in Fig. 1.

In this paper, sufficient conditions for an admittance to ensure force-guided assembly are established for each of these three PCs.

\section{SECTION II. Configuration Description}

In this section, the sets of coordinates used to describe configuration variation for single-point contact PCs are presented.



(a)

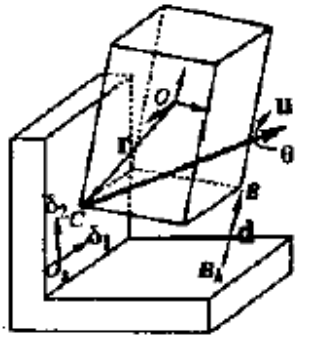

(b)

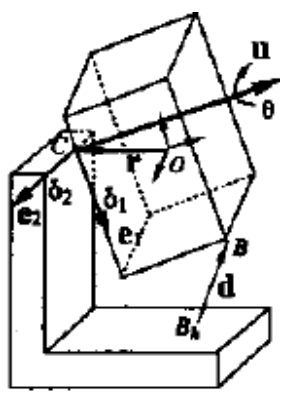

(c)

Fig. 1. Configuration Variables for Single-Point Principle Contacts. (a) Face-Vertex Contact. (b) Vertex-Face Contact. (c) EdgeEdge Cross Contact.

As previously stated, the three contact PCs are face-vertex, vertex-face and edge-edge cross contact (as shown in Fig. 1). The body can translate in the plane of contact and rotate about the contact point in any direction. As such, five variables describe the relative configuration of the bodies (the relative position of the contact point using two translational variables and the relative orientation using three rotational variables). 


\section{1) Orientational Variation}

The relative orientation of the rigid body can be described by a $3 \times 3$ orthogonal matrix $\mathbf{R}$.

Consider two configurations $C_{0}$ and $C_{1}$ with the same point of contact. By Euler theory, there exists an axis such that configuration $C_{1}$ can be achieved from configuration $C_{0}$ by a rotation about this single axis. For any given $C_{0}$ and $C_{1}$, the direction of the axis $u$ and rotation angle $\theta$ are unique $(0 \leq \theta \leq \pi)$.

Consider a rotation about an arbitrary axis u with angle $\theta$. The rotation matrix associated with this configuration change can be obtained by Rodrigues' formula: ${ }^{14}$

$$
\mathbf{R}(\mathrm{u}, \theta)=\mathbf{I}+(1-\cos \theta) \mathrm{uu} T+\sin \theta[\mathrm{u} \times](3)
$$

where $\left[\begin{array}{ll}u & x\end{array}\right]$ denotes the anti-symmetric matrix associated with the cross product operation involving $u$ given by:

$$
[\mathrm{u} \times]=\left[\begin{array}{ccc}
0 & -u_{3} & u_{2} \\
u_{3} & 0 & -u_{1} \\
-u_{2} & u_{1} & 0
\end{array}\right]
$$

Finite variation from an initial configuration (considered later in establishing sufficient conditions) can be described by: 1) placing bounds on the maximum angular magnitude $0 \leq \theta \leq \theta_{M}$ and 2) providing no bounds on the direction of the rotation axis, $u$.

Since the orientational error is bounded by possible manipulator inaccuracy within a specified contact state, only small angular variation $\left(\leq 10^{\circ}\right)$ is considered. Because $u$ is arbitrary, for a centered coordinate frame with maximum angular variation $\Delta \theta$, the bound for the angular magnitude $\theta_{M}=\frac{1}{2} \Delta \theta$. For example, if the maximum angular variation considered is $10^{\circ}<0$ then $\theta_{M}=5^{\circ}$.

\section{2) Translational Variation}

For bodies in contact at a single point, the location of the contact point can be described by two parameters $\delta=\left(\delta_{1}, \delta_{2}\right)$. The meaning of these variables is different for the different principle contacts.

For face-vertex $(\{f-v\})$ contact, a 2-dimensional coordinate frame $O_{b}$ is established on the held body in the plane of the contact face. Two orthogonal coordinates $\left(\delta_{1}, \delta_{2}\right)$ are used to describe translational variation of the rigid body within this contact state as shown in Fig. 1 a.

For vertex-face $(\{v-f\})$ contact, a 2-dimensional coordinate frame $O_{s}$ is established on the stationary part in the plane of the contact face. Again, two orthogonal coordinates $\left(\delta_{1}, \delta_{2}\right)$ are used to describe the translational variation of the rigid body within this contact state as shown in Fig. $1 \mathrm{~b}$. 
For edge-edge cross $\left(\{e-e\}_{c}\right)$ contact, two translational non-orthogonal coordinates $\left(\delta_{1}, \delta_{2}\right)$ are chosen to describe translational variation along edges $\mathrm{e}_{1}$ and $\mathrm{e}_{2}$ as shown in Fig. 1c.

Since finite configuration variation is considered, for each contact state, the variation of each $\delta_{i}$ is bounded. By appropriately choosing the coordinate origin (at a central location of contact), the bounds for $\delta_{i}$ can be written as:

$$
-\delta_{M_{i}} \leq \delta_{i} \leq \delta_{M_{i}}
$$

In summary, configuration variation for each single-point contact state is given by $\mathbf{q}=\left(\delta_{1}, \delta_{2}, \mathrm{u}, \theta\right)$.

\section{SECTION III. Error-Reducing Motion of a Constrained Rigid Body}

In this section, the motion of a partially constrained body is investigated. For single-point contact states, the contact force is imposed at the point of contact and is along the face normal (for ( $\{v-e\}$ and ( $\{e-$ $v\}$ contact states) or along the normal determined by the two contact edges (for $\{e-e\}_{c}$ contact).

Let $\mathrm{n}$ be a unit 3-vector indicating the direction of the normal contact force applied to the held body. The unit wrench associated with the normal force has the form:

$$
\mathrm{w}_{n}=\left[\begin{array}{c}
\mathrm{n} \\
\mathrm{r} \times \mathrm{n}
\end{array}\right](4)
$$

where $r$ is the position vector from the origin of the held body coordinate frame to the contact point, $C$, as shown in Fig. 1.

Let $\phi$ be the magnitude of the normal contact force. By the control law (1), the motion of the body is:

$$
\mathrm{v}=\mathrm{v}_{0}+\mathbf{A} \mathbf{w}_{n} \phi \cdot(5)
$$

Because the motion of the rigid body cannot penetrate the surface, the reciprocal condition ${ }^{15}$ must be satisfied:

$$
\mathbf{w}_{n}^{T} \mathbf{v}=\mathbf{w}_{n}^{T} \mathbf{v}_{0}+\mathbf{w}_{n}^{T} \mathbf{A} \mathbf{w}_{n} \phi=0
$$

Solving for $\phi$ in the above equation and substituting into (5) yields

$$
\mathbf{v}=\frac{\left(\mathbf{v}_{0} \mathbf{w}_{\mathrm{n}}^{T}-\mathbf{v}_{0}^{\mathrm{T}} \mathbf{w}_{\mathrm{n}} \mathrm{I}\right) \mathrm{A} \mathbf{w}_{n}}{\mathbf{w}_{\mathrm{n}}^{T} \mathrm{~A} \mathbf{w}_{n}}
$$

For the compliant motion to be error-reducing, condition (2) must be satisfied for a given point. Thus, (2) becomes:

$$
E=\frac{\mathbf{d}^{T}\left(\mathbf{v}_{0} \mathbf{w}_{n}^{T}-\mathbf{v}_{0}^{T} \mathbf{w}_{n} \mathbf{I}\right) \mathbf{A} \mathbf{w}_{n}}{\mathbf{w}_{n}^{T} \mathbf{A} \mathbf{w}_{n}}<0 \text { (7) }
$$

where $A, d$, and $\mathbf{w}_{n}$ are expressed in the held body frame. 


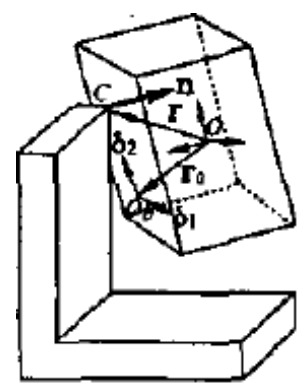

(a)

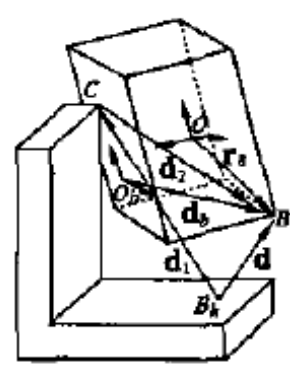

(b)

Fig. 2. Face-Vertex Contact. (a) Contact Force in the Body Frame. (b) Error-Measure Vector $\mathbf{d}$ in the Body Frame.

Since A is positive definite, $\mathbf{w}_{n}^{T} \mathbf{A} \mathbf{w}_{n}>0$, and the denominator of (7) is positive. Therefore, the errorreduction function can be expressed as:

$$
F_{e r}=\mathrm{d}^{T}\left(\mathrm{v}_{0} \mathrm{w}_{n}^{T}-\mathrm{v}_{0}^{T} \mathrm{w}_{n} \mathbf{I}\right) \mathbf{A} \mathbf{w}_{n} \cdot(8)
$$

Since $\mathbf{d}$ and $\mathrm{w}_{n}$ are functions of configuration $\mathrm{q}, F_{e r}$ is a function of $q$, To obtain error reducing motion, $F_{e r}(\mathrm{q})$ must be negative for all $q$ considered within the specified principle contact.

\section{SECTION IV. Conditions for Face-Vertex Contact}

As shown in Section II, the relative configuration of the bodies for face-vertex contact is described by the translation variables $\left(\delta_{1}, \delta_{2}\right)$ and orientational variables $(\mathrm{u}, \theta)$. We prove that, if an admittance matrix $\mathrm{A}$ satisfies a set of conditions at the "boundary" points, then the A matrix ensures error-reducing motion for all intermediate configurations $\delta_{i} \in\left[-\delta_{M_{i}}, \delta_{M_{i}}\right]$ and $\theta \in\left[0, \theta_{M}\right]$ (regardless of the direction of rotation).

\section{A. Error-Reduction Function}

For a face-vertex contact state as shown in Fig. 2a, when the held body rotates relative to the fixtured body about the contact point, the description of the contact wrench does not change in a body-based coordinate frame. When the held body translates relative to the fixtured body, the description of the contact wrench changes in a body-based coordinate frame because the contact point changes (although its direction is constant). Thus, the contact wrench is a function involving only the translational variables $\left(\delta_{1}, \delta_{2}\right)$.

For all face-vertex cases, the direction of the surface normal is constant in the body frame while the position vector of the contact point, $r$, varies. For arbitrary $\left(\delta_{1}, \delta_{2}\right), \mathbf{r}$ can be expressed as:

$$
\mathrm{r}=\mathrm{r}_{0}+\delta_{1} \mathrm{u}_{1}+\delta_{2} \mathrm{u}_{2}
$$

where $r_{0}$ is the position vector from the body frame's origin $O$ to the origin of the centrally located coordinate frame $O_{b}$, and $\mathrm{u}_{1}$ and $\mathrm{u}_{2}$ are unit vectors along the two axes of coordinate frame $O_{b}$ (constant in body frame).

The unit wrench corresponding to the surface normal, $\mathrm{w}_{n}$, can be obtained by (4). It can be seen that in the body frame, the direction of $\mathrm{w}_{n}$ is constant while the last component (the moment term) is a linear function of $\delta_{i}$. 
As shown in Fig. $2 b$, the line vector $\mathbf{d}$ associated with error reduction is also a function of configuration. For arbitrary $\left(\delta_{1}, \delta_{2}\right)$ with $\theta=0$, the error-measure 3-vector $\mathrm{d}^{\prime}$ is:

$$
\mathrm{d}^{\prime}(\delta)=\mathrm{d}_{1}^{\prime}+\mathrm{d}_{b}^{\prime}-\delta_{1} \mathbf{u}_{1}-\delta_{2} \mathbf{u}_{2}, \delta_{i} \in\left[-\delta_{M_{i}}, \delta_{M_{i}}\right]
$$

where $\mathrm{d}_{1}^{\prime}$ is the position vector from $B_{h}$ to the contact point $C$ (constant in global frame) and $\mathrm{d}_{b}$ is the position vector from the frame origin $O_{b}$ to point $B$ (constant in body frame).

For an arbitrary orientation $(\mathrm{u}, \theta)$ and $\delta_{i} \in\left[-\delta_{M_{i}}, \delta_{M_{i}}\right.$, the error-measure 3-vector $\mathrm{d}^{\prime}$ is a function of $(\mathrm{u}, \theta)$ and $\delta_{i}$ having the form:

$$
\mathrm{d}^{\prime}(\mathrm{u}, \theta, \delta)=\mathbf{R d}_{1}^{\prime}+\mathrm{d}_{b}^{\prime}-\delta^{\prime}(9)
$$

where $\mathbf{R}$ is the rotation matrix having the form of (3) and $\delta^{\prime}=\delta_{1} \mathrm{u}_{1}+\delta_{2} \mathrm{u}_{2}$.

The line vector associated with $\mathrm{d}^{\prime}$ can be calculated:

$$
\mathrm{d}(\delta, \theta)=\left[\begin{array}{c}
\mathrm{Rd}_{1}^{\prime} \\
\mathrm{r}_{B} \times \mathrm{Rd}_{1}^{\prime}
\end{array}\right]+\left[\begin{array}{c}
\mathrm{d}_{b}^{\prime} \\
\mathrm{r}_{B} \times \mathrm{d}_{b}^{\prime}
\end{array}\right]-\left[\begin{array}{c}
\delta^{\prime} \\
\mathrm{r}_{B} \times \delta^{\prime}
\end{array}\right]
$$

where $\mathrm{r}_{B}$ is the position vector from the body frame origin $O$ to the error measure point $B$ (constant in body frame).

Thus, for any intermediate configuration $\left(\delta_{1}, \delta_{2}, \theta\right)$, using (4) and (10), the error-reduction function $F_{e r}$ in (8) can be expressed as a function of $\left(\delta_{1}, \delta_{2}, \mathrm{n}, \theta\right)$.

Since only small orientational variation is considered, the angular magnitude $\theta$ is small $\left(\leq 5^{\circ}\right)$. Thus the rotation matrix $\mathbf{R}$ in (3) can be accurately approximated by:

$$
\mathbf{R}(\mathrm{n}, \theta)=\mathbf{I}+\sin \theta[\mathrm{u} \times]
$$

In the following, for an arbitrary wrench (6D line vector) $w$, we denote $w_{u}$ as the cross product operation of $u$ on w. i.e., if $w$ has the form:

$$
\mathrm{w}=\left[\begin{array}{c}
\mathrm{a} \\
\mathrm{r} \times \mathrm{a}
\end{array}\right], \text { then } \mathrm{w}_{\mathrm{u}}=\left[\begin{array}{c}
\mathrm{u} \times \mathrm{a} \\
\mathrm{r} \times(\mathrm{u} \times \mathrm{a})
\end{array}\right] .
$$

If we denote: $\mathrm{d}_{0}^{\prime}=\mathrm{d}_{1}^{\prime}+\mathrm{d}_{b}^{\prime}$, and

$$
\mathrm{d}_{0}=\left[\begin{array}{c}
\mathrm{d}_{0}^{\prime} \\
\mathrm{r}_{\mathrm{B}} \times \mathrm{d}_{0}^{\prime}
\end{array}\right], \delta=\left[\begin{array}{c}
\mathrm{d}^{\prime} \\
\mathrm{r}_{\mathrm{B}} \times \mathrm{d}^{\prime}
\end{array}\right],
$$

then, using (8), the error-reduction function can be approximated by:

$$
\begin{array}{r}
F_{\mathrm{e} r}(\delta, \theta)=\left(\mathrm{d}_{0}-\delta\right)^{T}\left(\mathrm{v}_{0} \mathrm{w}_{n}^{T}-\mathrm{v}_{0}^{\mathrm{T}} \mathrm{w}_{n} \mathbf{I}\right) \mathbf{A} \mathbf{w}_{n}+ \\
{\left[\mathbf{d}_{\mathrm{u}}^{T}\left(\mathrm{v}_{0} \mathrm{w}_{n}^{T}-\mathrm{v}_{0}^{T} \mathrm{w}_{n} \mathbf{I}\right) \mathbf{A} \mathrm{w}_{\mathrm{n}}\right] \sin \theta .}
\end{array}
$$


Since $u$ is a unit vector,

$$
\mathrm{d}_{\mathrm{u}}=\left\|\left[\begin{array}{c}
\mathrm{u} \times \mathrm{d}_{0}^{\prime} \\
\mathrm{r}_{B} \times\left(\mathrm{u} \times \mathrm{d}_{0}^{\prime}\right)
\end{array}\right]\right\| \leq\left\|\left[\begin{array}{c}
\mathrm{d}_{0}^{\prime} \\
\mathrm{r}_{B} \times \mathrm{d}_{0}^{\prime}
\end{array}\right]\right\|=\left\|\mathrm{d}_{0}\right\|
$$

Thus, the second term in (13),

$$
\left[\mathrm{d}_{\mathrm{u}}^{T}\left(\mathrm{v}_{0} \mathrm{w}_{\mathrm{n}}^{T}-\mathrm{v}_{0}^{T} \mathrm{w}_{n} \mathbf{I}\right) A \mathbf{w}_{n}\right] \sin \theta \leq M \sin \theta_{M}
$$

where $M=\left\|\mathrm{d}_{0}\right\|\left\|\left(\mathrm{v}_{0} \mathrm{w}_{n}^{\mathrm{T}}-\mathrm{v}_{0}^{T} \mathrm{w}_{n} \mathbf{I}\right) A \mathbf{w}_{n}\right\|$

Now consider the first term in (13):

$$
f=\left(\mathrm{d}_{0}+\delta\right)^{T}\left(\mathrm{v}_{0} \mathrm{w}_{n}^{T}-\mathrm{v}_{0}^{T} \mathrm{w}_{n} \mathbf{I}\right) \mathbf{A} \mathbf{w}_{n} .
$$

It can be seen that $f$ is a third order polynomial in $\delta_{1}$ and $\delta_{2}$. Consider the function constructed by

$$
F\left(\delta_{1}, \delta_{2}\right)=f+M \sin \theta_{M} \cdot(14)
$$

Then $F$ is a third order polynomial in $\delta_{1}$ and $\delta_{2}$, and for all intermediate configurations, $F_{\text {er }} \leq F\left(\delta_{1}, \delta_{2}\right)$.

\section{B. Sufficient Conditions for Error-Reduction}

The error-reduction condition requires that the error-reduction function in (13) must be negative in the range of configurations considered. In order to obtain sufficient conditions, we consider the "more positive" function defined in (14). The polynomial can be written in the form:

$$
\begin{gathered}
F\left(\delta_{1}, \delta_{2}\right)=f_{1} \delta_{1}^{3}+f_{2} \delta_{1}^{2} \delta_{2}+f_{3} \delta_{1} \delta_{2}^{2}+f_{4} \delta_{2}^{3}+f_{5} \delta_{1}^{2}+ \\
f_{6} \delta_{1} \delta_{2}+f_{7} \delta_{2}^{2}+f_{8} \delta_{1}+f_{9} \delta_{2}+f_{0}
\end{gathered}
$$

If $F_{e r}$ is negative for a configuration $\mathrm{q}_{0}$ and has no root for all $\delta_{1} \in\left[-\delta_{M_{1}}, \delta_{M_{1}}\right]$ and $\delta_{2} \in\left[-\delta_{M_{2}}, \delta_{M_{2}}\right]$, then the error-reduction condition is ensured.

Denote

$$
\begin{aligned}
& f_{\delta_{2}}=f_{4} \delta_{2}^{3}+f_{7} \delta_{2}^{2}+f_{9} \delta_{2}+f_{0}, \\
& f_{m}=\min _{\left|\delta_{2}\right| \leq \delta_{M_{2}}}\left\{\left|f_{\delta_{2}}\right|\right\}, \\
& c_{M}=\min _{\left|\delta_{2}\right| \leq \delta_{M_{2}}}\left\{\left|f_{1}\right|,\left|f_{2} \delta_{2}+f_{5}\right|,\left|f_{3} \delta_{2}^{2}+f_{6} \delta_{2}+f_{8}\right|\right\} .
\end{aligned}
$$

It can be proved that if 


$$
\frac{f_{m}}{c_{M}+f_{m}} \geq \delta_{M_{1}}
$$

then, $F_{e r}$ has no root for all $\delta_{i} \in\left[-\delta_{M_{i}}, \delta_{M_{i}}\right]$. Since $f_{m}$ in (17) and $c_{M}$ in (18) are functions of the admittance $\mathbf{A},(19)$ imposes a constraint on A. In summary, we have:

\section{Proposition 1}

For an face-vertex contact state, if: i) at the configuration $\left(\delta_{1}, \delta_{2}, \theta\right)=(0,0,0)$, the admittance satisfies the error reduction condition (2), and ii) condition (19) is satisfied for the polynomial (15), then the admittance will satisfy the error reduction conditions for all configurations bounded by $\delta_{i} \in$ $\left[-\delta_{M_{i}}, \delta_{M_{i}}\right]$ and $\theta \in\left[0, \theta_{M}\right]$ in any rotation direction.

Note that, since the functions in (16)-(18) are all polynomials in $\delta_{2}$ with order no higber than 3 , the maximum and minimum values of these functions can be obtained analytically by evaluating the function at the boundary points $\pm \delta_{M_{2}}$ and the stationary points. Thus, to ensure that contact yields error-reducing motion for the body for an face-vertex contact state, only two conditions [(2) and (19)] need be satisfied.

\section{SECTION V. Conditions for Vertex-Face Contact}

In this section, vertex-face contact is considered. As shown in Fig. 1b, the configuration of the body can be determined by the orientation of the body $(u, \theta)$ and the location of the contact point $\left(\delta_{1}, \delta_{2}\right)$.

Suppose that $\theta$ varies within the range of $\left[0, \theta_{M}\right]$, and $\delta_{i}$ varies within the range of $\left[-\delta_{M_{i}}, \delta_{M_{i}}\right]$. To obtain the error-reduction condition, we first consider configuration variation in orientation and translation separately. Then, by combining the two cases, general results are obtained.

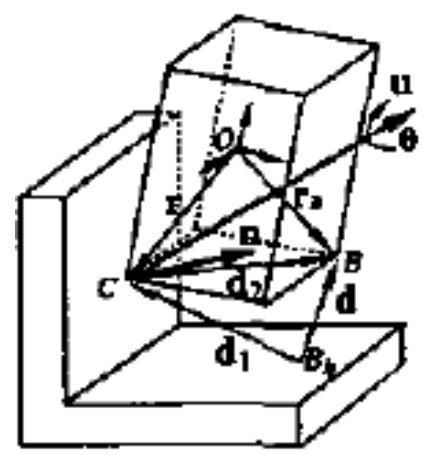

(a)

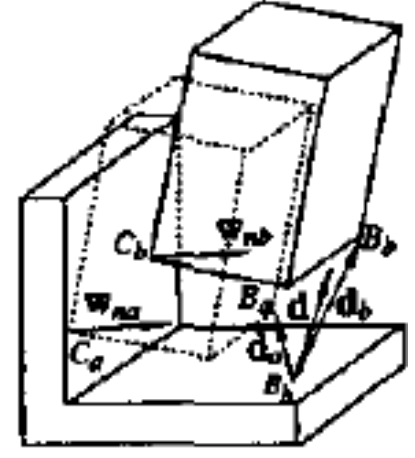

(b)

Fig. 3. Vertex-Face Contact State. (a) Orientational Variation. (b) Translational Variation,

\section{A. Configuration Variation in Orientation}

Consider only orientational variation of the contact configuration as illustrated in Fig. $3 a$. In this case, the location of the contact vertex of the held body is constant in the face plane, and both the direction of the error reduction vector $\mathbf{d}$ and the direction of the contact force are changed by changing the orientation, We prove that, for $\theta_{M} \leq \frac{\pi}{10}$, if $\mathrm{A}$ satisfies a set of conditions at $\theta=0$ (defined at a central orientation), then an error-reducing motion is ensured for all configurations obtained by rotating about an arbitrary axis $\mathbf{u}$ with angle $\theta \leq \theta_{M}$. 


\section{1) Error Reduction Function}

Let $\mathbf{w}_{n 0}$ be the wrench, and $\mathrm{d}_{0}$ be the position vector associated with $\theta=0$, Consider a rotation given by an angle change $\theta \in\left[0, \theta_{M}\right]$ about an axis $\mathbf{u}$, If we denote $\mathrm{n}_{0}$ as the surface normal associated with $\theta=$ 0 , then in the body coordination frame, the surface normal associated with varying $(\mathrm{u}, \theta)$ is: $\mathrm{n}_{\theta}=$ $\mathrm{R}(\theta) \mathrm{n}_{0}$, where $\mathbf{R}$ is the rotation matrix having the form of (11).

Since contact is frictionless, the contact force is along the surface normal at the contact point. Thus, the unit contact wrench is:

$$
\mathrm{w}_{n}(\theta)=\left[\begin{array}{c}
\mathrm{n}_{\theta} \\
\mathrm{r} \times \mathrm{n}_{\theta}
\end{array}\right]=\left[\begin{array}{c}
\mathrm{Rn}_{0} \\
\mathrm{r} \times \mathrm{Rn}_{0}
\end{array}\right](20)
$$

where $r$ is the position vector from the origin of the body frame to the contact point (constant in body frame).

Since the orientational variation considered corresponds to pure rotation about the contact point, the error-measure 6-vector $d$ for an intermediate configuration can be expressed in the body frame as:

$$
\mathrm{d}(\theta)=\left[\begin{array}{c}
\mathrm{d}^{\prime} \\
\mathrm{r}_{B} \times \mathrm{d}^{\prime}
\end{array}\right]=\left[\begin{array}{c}
\mathrm{Rd}_{1}^{\prime} \\
\mathrm{r}_{B} \times \mathrm{Rd}_{1}^{\prime}
\end{array}\right]+\left[\begin{array}{c}
\mathrm{d}_{2}^{\prime} \\
\mathrm{r}_{B} \times \mathrm{d}_{2}^{\prime}
\end{array}\right]
$$

where $\mathrm{d}_{1}^{\prime}$ is the position 3-vector from $B_{h}$ to the contact point $C, \mathrm{~d}_{2}^{\prime}$, is the position 3-vector from $C$ to point $B$ and $r_{B}$ is the position vector from the body frame origin to point $B$.

Substituting (20) and (21) into (8) and using (11), the error-reduction function can be expressed as a function of $(u, \theta)$ in the form:

$$
F_{e r}(\theta)=F_{\mathrm{e} r}(0)+F_{1} \sin \theta+F_{2} \sin ^{2} \theta+F_{3} \sin ^{3} \theta,(22)
$$

where

$$
\begin{aligned}
& F_{\mathrm{I}}=\mathrm{d}_{0}^{\mathrm{T}}\left(\mathrm{v}_{0} \mathrm{w}_{0}^{T}-\mathrm{v}_{0}^{T} \mathrm{w}_{0} \mathbf{I}\right) A \mathrm{w}_{\mathrm{u}}-\mathrm{d}_{0}^{\mathrm{T}}\left(\mathrm{v}_{0} \mathrm{w}_{\mathrm{u}}^{T}-\mathrm{v}_{0}^{T} \mathrm{w}_{\mathrm{u}} \mathbf{I}\right) A \mathrm{w}_{0} \\
& -\mathrm{d}_{\mathrm{u}}^{T}\left(-\mathrm{v}_{0} \mathrm{w}_{0}^{T}+\mathrm{v}_{0}^{T} \mathrm{w}_{0} \mathbf{I}\right) A \mathrm{w}_{0} \\
& F_{2}=\mathrm{d}_{0}^{\mathrm{T}}\left(\mathrm{v}_{0} \mathrm{w}_{\mathrm{u}}^{T}-\mathrm{v}_{0}^{\mathrm{T}} \mathrm{w}_{\mathrm{u}} \mathbf{I}\right) A \mathbf{w}_{\mathrm{u}}+\mathrm{d}_{\mathrm{u}}^{T}\left(\mathrm{v}_{0} \mathrm{w}_{\mathrm{u}}^{T}-\mathrm{v}_{0}^{T} \mathrm{w}_{\mathrm{u}} \mathbf{I}\right) A \mathrm{w}_{0} \\
& +\mathrm{d}_{\mathrm{u}}^{T}\left(\mathrm{v}_{0} \mathrm{w}_{0}^{T}-\mathrm{v}_{0}^{T} \mathrm{w}_{0} \mathbf{I}\right) A \mathbf{w}_{\mathrm{u}} \\
& F_{3}=\mathrm{d}_{\mathrm{u}}^{\mathrm{T}}\left(-\mathrm{v}_{0} \mathrm{w}_{\mathrm{u}}^{T}+\mathrm{v}_{0}^{T} \mathrm{w}_{\mathrm{u}} \mathbf{I}\right) A \mathbf{w}_{\mathrm{u}},
\end{aligned}
$$

where the subscript $u$ of a wrench indicates the cross product operation of $u$ on the wrench [as defined in (12)]. 


\section{2) Error Reduction Conditions}

To achieve error reduction at all other configurations considered, $F_{e r}(\theta)$ must be negative for $\theta \in$ $\left[0, \theta_{M}\right]$ and an arbitrary rotation axis $u$. Since $u$ is a unit vector, the bounds for $F_{i}$ in $(22)$ can be obtained.

If we denote: $M=\left|\mathrm{d}_{0}\right| \cdot\left\|\left(\mathrm{v}_{0} \mathrm{w}_{0}^{T}-\mathrm{v}_{0}^{T} \mathrm{w}_{0} \mathrm{I}\right) \mathbf{A}\right\| \cdot \mid \mathrm{w}_{0}$, then, $\left|F_{1}\right| \leq 3 M,\left|F_{2}\right| \leq 3 M,\left|F_{3}\right| \leq M$. Consider the new function constructed by:

$$
F=F_{\mathrm{e} r}(0)+3 M \sin \theta_{M}+3 M \sin ^{2} \theta_{M}+M \sin ^{3} \theta_{M}
$$

Then, for $\theta \in\left[0, \theta_{M}\right] s$ with an arbitrary rotation axis, we have $F_{\text {er }}(\mathrm{u}, \theta) \leq F$. Thus, if

$$
F_{\mathrm{er}}(0\rangle+3 M \sin \theta_{M}+3 M \sin ^{2} \theta_{M}+M \sin ^{3} \theta_{M}<0,(23)
$$

then $F_{\text {er }}(\mathrm{u}, \theta)<0$ for all orientational variations considered.

\section{B. Configuration Variation in Translation}

Now consider the translational variation of the contact configuration illustrated in Fig. $3 \mathrm{~b}$. In this case, only translation of the contact point in the contact face is allowed, and the contact force does not change in the body frame. The procedure used in the planar vertex-face contact ${ }^{9}$ can be extended to this case. Thus, if at two configurations $\left(-\delta_{M_{1}}, \delta_{2}\right)$ and $\left(\delta_{M_{1}}, \delta_{2}\right)$ the error reduction condition is satisfied, then the error reduction condition must be satisfied for all intermediate configurations $\left(\left(\delta_{1}, \delta_{2}\right)\right.$ with $\delta_{1} \in\left[-\delta_{M_{1}}, \delta_{M_{1}}\right]$. The same result holds true for variation in $\delta_{2}$ while $\delta_{1}$ is-constant.

\section{General Case}

Using the process used for the planar case, the results presented in V-A and V-B can be generalized to intermediate vertex-face contact configurations involving both translational and orientational variations from configurations at which the conditions were imposed. Thus we have:

\section{Proposition 2}

For a vertex-face contact state with variation of orientation $0, \theta_{M}$ and variation of translation $\left[-\delta_{M_{i}} \delta_{M_{i}}\right]$, if inequality (23) is satisfied at the four translational boundary points $\left( \pm \delta_{M_{1}}, \pm \delta_{M_{2}}\right)$, then the admittance will satisfy the error reduction condition for all configurations bounded by $\delta_{i} \in\left[-\delta_{M_{i}}, \delta_{M_{i}}\right]$, and $\theta \in$ $\left[0, \theta_{M}\right]$ in any rotation direction.

Thus, for a face-vertex contact state; to ensure that the motion response due to contact is error reducing for all configurations considered, only four conditions need be satisfied. 


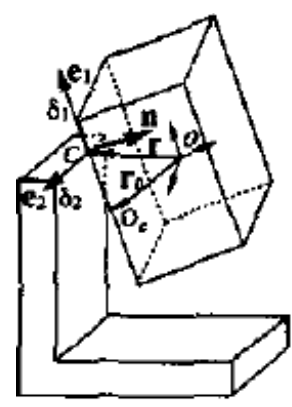

(a)

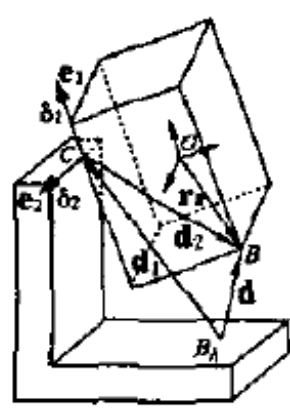

(b)

Fig. 4. Edge-Edge Cross Contact. (A) Contact Force in the Body Frame. (b) Error-Measure Vector d in the Body Frame.

\section{SECTION VI. Conditions for Edge-edge Cross Contact}

Below, for "edge-edge cross" contact, we identify the set of conditions that, when satisfied for a given admittance matrix $A$ at the "boundary" points, ensures error-reducing motion for all intermediate configurations $\theta \in\left[0, \theta_{M}\right], \delta_{i} \in\left[-\delta_{M_{i}}, \delta_{M_{i}}\right]$.

\section{A. Error-Reduction Function}

For an edge-edge cross contact state as shown in Fig. 4a, the direction of the contact force is along the common normal of the two edges. Let $\mathrm{e}_{1}$ and $\mathrm{e}_{2}$ be the two unit vectors along the two edges respectively, then the direction of the force must be $\mathrm{n}=\mathrm{e}_{1} \times \mathrm{e}_{2}$. Note that $e_{1}$ is constant in the body frame while $\mathrm{e}_{2}$ is constant in the global frame. When the held body rotates relative to the fixtured body about the contact point $C$, the vector $\mathrm{e}_{2}$ in the body frame can be expressed as $\operatorname{Re}_{2}$ where $\mathbf{R}$ is the rotation matrix. When the held body translates relative to the fixtured body along $\mathrm{e}_{1}$ as shown in Fig. $4 \mathrm{a}$, the description of the contact wrench changes in a body-based coordinate frame as the contact point changes (although its direction is constant). Thus, the contact wrench is a function involving both the translational and orientational variables $\left(\delta_{1}, \delta_{2}, \theta\right)$

For all edge-edge cross contact cases, the direction of the force depends only on the orientational variation while the position vector of the contact point, $r$, depends only the translational variation along the contact edge of the held body $\mathrm{e}_{1}$. For arbitrary $\left(\delta_{1}, \delta_{2}\right), \mathbf{r}$ can be expressed as: $\mathrm{r}_{\delta}=\mathbf{r}_{0}+\delta_{1} \mathbf{e}_{1}$, where $\mathrm{r}_{0}$ is a vector from the body frame to a centrally located point on the edge $\mathrm{e}_{1}$ (constant).

For rotation $\mathbf{R}$ the direction of the normal force is: $n=e_{1} \times \operatorname{Re}_{2}$. By (4), the unit contact wrench can be expressed as:

$$
\mathrm{w}_{n}=\left[\begin{array}{c}
\mathrm{e}_{1} \times \mathrm{Re}_{2} \\
\mathrm{r}_{\delta} \times\left(\mathrm{e}_{1} \times \mathrm{Re}_{2}\right)
\end{array}\right]
$$

Let $\mathrm{d}_{1}^{\prime}$ and $\mathrm{d}_{2}^{\prime}$ be the two vectors from $B_{h}$ to $C$ and from $C$ to $B$ for $(\delta, \theta)=(0,0)$ respectively, and let $d_{1}$ and $d_{2}$ be the corresponding line vector associated with vector $r_{B}$ (as shown in Fig. $4 b$ ), then for an arbitrary orientation $(\mathrm{u}, \theta)$ and $\delta_{i} \in\left[-\delta_{M_{i}}, \delta_{M_{i}}\right]$, the error-measure line-vector $\mathrm{d}$ can be expressed as:

$$
\mathrm{d}=\mathrm{R}\left(\mathrm{d}_{1}+\delta_{2}\right)+\mathrm{d}_{2}+\delta_{1}
$$


where

$$
\delta_{1}=\delta_{1}\left[\begin{array}{c}
\mathrm{e}_{1} \\
\mathrm{r}_{B} \times \mathrm{e}_{1}
\end{array}\right], \delta_{2}=\delta_{2}\left[\begin{array}{c}
\mathrm{e}_{2} \\
\mathrm{r}_{B} \times \mathrm{e}_{2}
\end{array}\right]
$$

For small $\theta$, the expression of $\mathbf{R}$ in (11) provides an accurate approximation. Substituting the above $\mathrm{w}_{n}$ and $d$ into (8) and sorting the coefficients of $\sin \theta$, the error-reduction function can be expressed as:

$$
F_{e r}(\delta, \theta)=F_{0}+F_{1} \sin \theta+F_{2} \sin ^{2} \theta+F_{3} \sin ^{3} \theta,
$$

where:

$$
\begin{aligned}
& F_{0}=\left(\mathrm{d}_{1}+\mathrm{d}_{2}+\delta_{1}+\delta_{2}\right)^{T}\left(\mathrm{~V}_{0} \mathrm{w}_{0}^{T}-\mathrm{v}_{0}^{\mathrm{T}} \mathrm{w}_{0} \mathrm{I}\right) A \mathrm{w}_{0}, \\
& F_{1}=-\left(\mathrm{d}_{1}+\mathrm{d}_{2}+\delta_{1}+\delta_{2}\right)^{\mathrm{T}}\left(\mathrm{v}_{0} \mathrm{w}_{\mathrm{u}}^{\mathrm{T}}-\mathrm{v}_{0}^{\mathrm{T}} \mathrm{w}_{\mathrm{u}} \mathrm{I}\right) A \mathrm{w}_{0} \\
& -\left(\mathrm{d}_{1}+\mathrm{d}_{2}+\delta_{1}+\delta_{2}\right)^{T}\left(\mathrm{v}_{0} \mathrm{w}_{0}^{T}-\mathrm{v}_{0}^{\mathrm{T}} \mathrm{w}_{0} \mathrm{I}\right) A \mathrm{w}_{\mathrm{u}} \\
& +\left(\mathrm{d}_{1}+\delta_{2}\right)_{\mathrm{u}}^{T}\left(\mathrm{v}_{0} \mathrm{w}_{0}^{\mathrm{T}}-\mathrm{v}_{0}^{T} \mathrm{w}_{0} \mathrm{I}\right) A \mathrm{w}_{0} \\
& +\left(\mathrm{d}_{2}+\delta_{1}\right)^{T}\left(\mathrm{v}_{0} \mathrm{w}_{0}^{T}-\mathrm{v}_{0}^{T} \mathrm{w}_{0} \mathrm{I}\right) A \mathrm{w}_{\mathrm{u}} \\
& -\left(\mathrm{d}_{1}+\delta_{1}\right)^{T}\left(\mathrm{v}_{0} \mathrm{w}_{\mathrm{u}}^{\mathrm{T}}-\mathrm{v}_{0}^{T} \mathrm{w}_{\mathrm{u}} \mathrm{I}\right) A \mathrm{w}_{0}, \\
& F_{2}=\left(\mathrm{d}_{1}+\delta_{2}\right)_{\mathrm{u}}^{T}\left(\mathrm{v}_{0} \mathrm{w}_{\mathrm{u}}^{T}-\mathrm{v}_{0}^{T} \mathrm{w}_{\mathrm{u}} \mathbf{I}-\mathrm{v}_{0} \mathrm{w}_{\mathrm{u}}^{T}+\mathrm{v}_{0}^{T} \mathrm{w}_{\mathrm{u}} \mathbf{I}\right) A \mathrm{w}_{\mathrm{u}} \\
& +\left(\mathrm{d}_{1}+\mathrm{d}_{2}+\delta_{1}+\delta_{2}\right)^{\mathrm{T}}\left(\mathrm{v}_{0} \mathrm{w}_{\mathrm{u}}^{\mathrm{T}}-\mathrm{v}_{0}^{T} \mathrm{w}_{\mathrm{u}} \mathbf{I}\right) A \mathrm{w}_{\mathrm{u}}, \\
& F_{3}=\left(\mathrm{d}_{1}+\delta_{2}\right)_{\mathrm{u}}^{\mathrm{T}}\left(\mathrm{v}_{0} \mathrm{w}_{\mathrm{u}}^{T}-\mathrm{v}_{0}^{T} \mathrm{w}_{\mathrm{u}} \mathrm{I}\right) A \mathrm{w}_{\mathrm{u}},
\end{aligned}
$$

where wo is the unit wrench when $\theta=0$ and the subscript $u$ of a wrench indicates the cross product operation of $u$ on the wrench [as defined in (12)].

Similar to the results presented in V-A.2, because $u$ is a unit vector, each $F_{i}$ in the above equation is bounded. If we denote $F_{M_{i}}=\max \left\{\left|F_{i}\right|\right\}(i=1,2,3)$, and consider the function defined by:

$$
F=F_{0}+F_{M_{1}} \sin \theta_{M}+F_{M_{2}} \sin ^{2} \theta_{M}+-F_{M_{3}} \sin ^{3} \theta_{M},(24)
$$

then, $F$ is a linear function in $\delta_{1}$ and $\delta_{2}$, and for all $\delta_{i} \in\left[-\delta_{M_{i}}, \delta_{M_{i}}\right]$ and $\theta \in\left[0, \theta_{M}\right], F_{e r}<F$.

Thus, if $F$ is negative for $\delta_{i} \in\left[-\delta_{M_{i}}, \delta_{M_{i}}\right]$, then $F_{\text {er }}$ must be negative for $\delta_{i} \in\left[-\delta_{M_{i}}, \delta_{M_{i}}\right]$ and for all rotations with $\theta \leq \theta_{M}$ in any direction. Since $F$ is a linear function in $\delta_{1}$ and $\delta_{2}, F<0$ for all $\delta_{i}$ 'S in the bounded area if and only if, at the four extremal points $\left( \pm \delta_{M_{1}}, \pm \delta_{M_{2}}\right), F<0$. Thus, we have:

\section{Proposition 3}

For a edge-edge cross contact state with variation of orientation $\left[0, \theta_{M}\right]$ and variation of translation $\left[-\delta_{M_{i}}, \delta_{M_{i}}\right]$, if at the four boundary points $\left( \pm \delta_{M_{1}}, \pm \delta_{M_{2}}\right)$. the function $F$ defined in (24) is negative, then the admittance will satisfy the error reduction condition for all configurations bounded by $\delta_{i} \in\left[-\delta_{M_{i}}, \delta_{M_{i}}\right]$ and rotation in an arbitrary direction with angle $\theta \leq \theta_{M}$. 


\section{SECTION VII. Discussion and Summary}

In this paper, sufficient conditions for spatial error-reducing motion of a held body are identified. Finite misalignment is considered when evaluating error-reduction. The bounds of errors considered are based on the maximum translational range of the body and the maximum magnitude of the body's Euler rotation (regardless of the direction). The error-reduction measure is based on a single point on the held body, If that point corresponds to that which is maximally displaced from its proper position, an established metric $^{12}$ is used as a measure of error-reduction. Alternately, the results could be applied to a finite set of points to further restrict the description of error-reduction.

In robotic application, the orientational misalignment due to the manipulator'S inaccuracy is small. Thus, the orientational variation considered is small (approximately $\pm 5^{\circ}$ ). For this range the approximated rotation matrix in (11) is accurate. Also, to obtain sufficient conditions for each contact state, conservative bounds on functions for translational and orientational variations are used. Thus, the sufficient conditions obtained are conservative for all contact states.

In summnary, we have presented an approach for admittance selection of a polyhedral rigid body for forceguided assembly. We have shown that, for single-point contact cases, the admittance control law can be selected based on their behavior at a finite number of configurations. If the error reduction conditions are satisfied at these configurations, the error reduction conditions will be satisfied for all intermediate configurations.

\section{References}

1. J. M. Schimmels, M. A. Peshkin, "Admittance matrix design for force guided assembly", IEEE Transactions on Robotics and Automation, vol. 8, no. 2, pp. 213-227, 1992.

2. D. E. Whitney, "Force feedback control of manipulator fine motions", ASME Journal of Dynamic Systems. Measurement. and Control, vol. 99, no. 2, pp. 91-97, 1977.

3. D. E. Whitney, "Quasi-static assembly of compliantly supported rigid parts", ASME Journal of Dynamic Systems Measurement and Control, vol. 104, no. 1, pp. 65-77, 1982.

4. M. A. Peshkin, "Programmed compliance for error-corrective manipulation", IEEE Transactions on Robotics and Automation, vol. 6, no. 4, pp. 473-482, 1990.

5. E. D. Fasse, J. F. Broenink, "A spatial impedance controller for robotic manipulation", IEEE Transactions on Robotics and Automation, vol. 13, no. 4, pp. 546-556, 1997.

6. J. Marcelo, H. Ang, G. B Andeen, "Specifying and achieving passive compliance based on manipulator structure", IEEE Transactions on Robotics and Automation, vol. 11, no. 4, pp. 504-515, 1995.

7. J. M. Schirnmels, M. A. Peshkin, "Force-assembly with friction", IEEE Transactions on Robotics and Automation, vol. 10, no. 4, pp. 465-479, 1994.

8. J. M. Schimmels, "A linear space of admittance control laws that guarantees force-assembly with friction", IEEE Transactions on Robotics and Automation, vol. 13, no. 5, pp. 656-667, 1997.

9. S. Huang, J. M. Schimmels, "Sufficient conditions used in admittance selection for force-guided assembly of polygonal parts", IEEE Transactions on Robotics and Automation, vol. 19, no. 4, pp. 737-742, 2003. 
10. S. Huang, J. M. Schimmels, "Admittance selection for planar force-guided assembly for single-point contact with friction", Proceeding of the IEEE International Conference on Robotics and Automation, vol. 3, pp. 3082-3088, September 2003.

11. S. Huang, J. M. Schimmels, "Sufficient conditions for admittance to ensure planar force-assembly in multi-point frictionless contact", Proceeding of the IEEE International Conference on Robotics and Automation, vol. 3, pp. 3095-3100, September 2003.

12. J. M. R. Martinez, J. Duffy, "On the metric of rigid body displacements for infinite and finite bodies", ASME Journal of Mechanical Design, vol. 117, no. 1, pp. 41-471, 1995.

13. J. Xiao, L. Zhang, "Contact constraint analysis and determination of geometrically valid contact formations from possible contact primitive", IEEE Transactions on Robotics and Automation, vol. 13, no. 3, pp. 456-466, 1997.

14. R. M. Murray, Z. Li, S. S. Sastry, A Mathematical Introduction to Robotic Manipulation, CRC Press, 1994.

15. M. S. Ohwovoriole, B. Roth, "An extension of screw theory", ASME Journal of Mechanical Design, vol. 103, no. 4, pp. 725-735, 1981. 